Pacific Journal of Mathematic 


\section{AN EXTENSION OF HAIMO'S FORM OF HANKEL CONVOLUTIONS}

\section{J. N. PANDEY}

The real inversion formula for Hankel convolutions which is due to D. T. Haimo, is extended to certain generalized functions. That is accomplished by transferring the real inversion formula of $D$. $T$. Haimo onto the testing function space for the generalized function under consideration and then showing that the limiting process in the resulting formula converges with respect to the topology of the testing function space.

The Hirschman-Widder convolution transformation [3] has recently been extended to certain classes of generalized functions [7], [4] and their inversion formulae [3; pp. 127-132 and Theorems 7.1 b, p. 231] have been shown to be still valid when the limiting operation in those formulae is understood as weak convergence in the space $D^{\prime}$ of Schwartz distributions [5]. The purpose of the present work is to extend the inversion formula of D. T. Haimo for Hankel convolutions [1, p. 332] in a similar way to a certain space of generalized functions.

The notation and terminology of this work follows that of [7], [4]. $I$ denotes the open interval $(0, \infty)$ and all testing functions herein are defined on $I$. Throughout this work $x$ and $y$ are variables over $I$ unless otherwise mentioned. Finally, $D(I)$ is the space of smooth functions defined on $I$ having compact supports. The topology of $D(I)$ is that which makes its dual the space $D^{\prime}(I)$ of Schwartz distributions [5; Vol. I, p. 65] [8] on $I$. Thus, a sequence of functions $\left\{\phi_{\nu}\right\}_{\nu=1}^{\infty}$ is said to converge in $D(I)$ if and only if the supports of $\phi_{\nu}$ are all contained within a fixed compact subset of $I$ and for each $k,\left\{\phi_{\nu}^{(k)}\right\}_{\nu=1}^{\infty}$ converges uniformly on $I$.

2. The testing function space $\mathscr{G}(I)$. Let $\Delta_{x}$ stand for the operator $\left(D_{x}^{2}+(2 \gamma / x) D_{x}\right)$ where $D_{x}$ is the differentiation operator and $\gamma$ is a positive number. We say that a smooth function $\phi(x)$ defined over $I$ belongs to $\mathscr{C}(I)$ if

$$
\gamma_{k}(\phi)=\sup _{0<x<\infty}\left|e^{c x} \Delta_{x}^{(k)} \phi(x)\right|<\infty
$$

for all $k$ assuming values $0,1,2, \ldots$. Here, $c$ is a fixed real number; but in our later discussion we will choose $c$ to be a fixed real number less than $a_{1}$. The topology in $\mathscr{C}(I)$ is generated by the collection of seminorms $\left\{\gamma_{k}\right\}_{k=0}^{\infty}$. Since $\gamma_{0}$ is a norm the collection of seminorms 
$\left\{\gamma_{k}\right\}_{k=0}^{\infty}$ is separating. We say that a sequence $\left\{\phi_{\nu}\right\}_{\nu=1}^{\infty}$ converges to $\phi$ in $\mathscr{G}(I)$ if for each $\phi_{\nu} \in \mathscr{C}(I)$ and for every fixed $k, \gamma_{k}\left(\phi_{\nu}-\phi\right)$ goes to zero as $\nu$ goes to $\infty . \mathscr{C}(I)$ is a locally convex Hausdorff topological linear space. $D(I)$ is a subspace of $\mathscr{G}(I)$ and the topology of $D(I)$ is stronger than the topology induced on $D(I)$ by $\mathscr{C}(I)$. Consequently the restriction of any member of $\mathscr{G}^{\prime}(I)$ to $D(I)$ is in $D^{\prime}(I)$.

DeFinition: We say that a sequence $\left\{\phi_{\nu}(x)\right\}_{\nu=1}^{\infty}$ where each $\phi_{\nu}(x) \in G(I)$ is a Cauchy sequence in $G(I)$ if $\gamma_{k}\left(\phi_{\nu}-\phi_{\mu}\right) \rightarrow 0$ as $\nu$ and $\mu$ both go to $\infty$ independently where $k$ assumes values $0,1,2,3, \cdots$.

LeMma 1. $\mathscr{C}(I)$ is sequentially complete.

Proof. By hypothesis the sequences

$$
\begin{array}{ll}
\left\{\phi_{\nu}(x)\right\}_{\nu=1}^{\infty}, & \left\{\int_{a}^{x} \phi_{\nu}(t) d t\right\}_{\nu=1}^{\infty} \\
\left\{\Delta_{x} \phi_{\nu}(x)\right\}_{\nu=1}^{\infty}, & \left\{\int_{a}^{x}\left[\Delta_{t} \dot{\phi}_{\nu}(t)\right] d t\right\}_{\nu=1}^{\infty}
\end{array}
$$

and $\left\{\int_{a}^{x} \int_{a}^{y}\left[\Delta_{t} \phi_{\nu}(t)\right] d t d y\right\}_{\nu=1}^{\infty}$ converge uniformly with respect to $x$ over any compact subset of $I$. We assume that $a$ is a fixed positive quantity. All these combined together mean that the sequence $\left\{\phi_{\nu}^{(k)}(x)\right\}_{\nu=1}^{\infty}$ for all $k$ converges uniformly over any compact subset of $I$ where $k$ assumes values $0,1,2$. Proceeding in the same way we can prove by induction that the sequence $\left\{\phi_{\nu}^{(k)}(x)\right\}_{\nu=1}^{\infty}$ for all $k$, where $k$ assumes values 0,1 , $2,3,4, \cdots$ converges uniformly over any compact subset of $I$. Here, uniformity is assumed with respect to the variable $x$ and not with respect to $k$. Therefore, by a classic result it follows that there exists a smooth function $\phi(x)$ such that the sequence $\left\{\phi_{\nu}^{(k)}(x)\right\}_{\nu=1}^{\infty}$ converges uniformly to $\dot{\phi}^{(k)}(x)$ on every compact subset of $I$ for $k=0,1$, $2, \cdots$. Our object is now to show that $\phi(x) \in \mathscr{C}(I)$. By hypothesis for $\varepsilon>0 \exists N$.

$$
\gamma_{k}\left(\phi_{\nu}-\phi_{\mu}\right)<\varepsilon \quad \text { for all } \nu>N, \mu>N .
$$

As $\mu \rightarrow \infty$ (2) reduces to

$$
\gamma_{k}\left(\dot{\phi}_{\nu}-\dot{\phi}\right)<\varepsilon \quad \text { for all } \nu>N .
$$

Now,

$$
\gamma_{k}(\dot{\phi})=\gamma_{k}\left(\dot{\phi}-\phi_{\nu}+\phi_{\nu}\right) \leqq \gamma_{k}\left(\phi_{\nu}-\dot{\phi}\right)+\gamma_{k}\left(\dot{\phi}_{\nu}\right) \text {. }
$$

By fixing a value of $\nu$ greater than $N$, (4) reduces to

$$
\gamma_{k}(\phi)<\varepsilon+\gamma_{k}\left(\phi_{\nu}\right)=\beta<\infty
$$




$$
\therefore \phi \in \mathscr{G}(I) \text {. }
$$

This completes the proof of our lemma.

3. The testing function space $\mathscr{H}(I)$. We say that a smooth function $\psi(x)$ defined on $I, \in \mathscr{C}(I)$ if $\left\{\psi(x) / \mu^{\prime}(x)\right\} \varepsilon \mathscr{G}(I)$ where $\mu(x)$ is the same as will be defined in (7). The topology in $\mathscr{C}(I)$ is generated by a sequence of seminorms $\left\{\beta_{k}\right\}_{k=0}^{\infty}$ where,

$$
\beta_{k}\{\psi(x)\}=\gamma_{k}\left\{\frac{\psi(x)}{\mu^{\prime}(x)}\right\}
$$

The concept of convergence and completeness in $\mathscr{C}(I)$ is defined in a way similar to that in $\mathscr{G}(I)$. $\mathscr{C}(I)$ is also a sequentially complete, Hausdorff, locally convex, topological linear space. $D(I)$ is a subspace of $\mathscr{C}(I)$ and restriction of any member of $\mathscr{C}^{\prime}(I)$ to $D(I)$ is in $D^{\prime}(I)$. The mapping $\psi(x) \rightarrow \psi(x) / \mu^{\prime}(x)$ in (6) is an isomorphism from $\mathscr{C}(I)$ onto $\mathscr{G}(I)$. It can be further proved that if $f \in \mathcal{P}^{\prime}(I)$ then $\mu^{\prime} f \in \mathscr{G}^{\prime}(I)$ and vice-versa [9, p. 28].

4. The generalized Hankel convolution of Haimo's type. Let us first specify the type of kernel for which our Hankel convolution has been constructed. We assume $\gamma$ to be a fixed positive number, and set as in [1],

$$
\begin{gathered}
\mu(x)=\frac{x^{2 \gamma+1}}{2^{\gamma+1 / 2} \Gamma\left(\gamma+\frac{3}{2}\right)} \\
J(x)=2^{\gamma-1 / 2} \Gamma\left(\gamma+\frac{1}{2}\right) x^{1 / 2-\gamma} J_{\gamma-1 / 2}(x),
\end{gathered}
$$

where $J_{\gamma-1 / 2}(x)$ is the ordinary Bessel function of order $(\gamma-1 / 2)$.

$$
G(x, y)=\int_{0}^{\infty} \frac{J(x t) J(y t) d \mu(t)}{\prod_{k=1}^{\infty}\left(1+\frac{t^{2}}{a_{k}^{2}}\right)}
$$

the $a_{k}$ 's being real, satisfying $0<a_{1} \leqq a_{2} \leqq a_{3} \cdots$ with

$$
\begin{gathered}
\sum_{k=1}^{\infty} a_{k}^{-2}<\infty \\
G_{N}(x, y)=\int_{0}^{\infty} \frac{J(x t) J(y t) d \mu(t)}{\prod_{k=N+1}^{\infty}\left(1+\frac{t^{2}}{a_{k}^{2}}\right)}
\end{gathered}
$$


$G(x, y)$ will be the kernel of Haimo's type of generalized Hankel convolutions. We briefly review some of the paramount properties of the kernel $G(x, y)$.

$$
\text { A. } \frac{\partial^{m+n}}{\partial x^{m} \partial y^{n}} G(x, y), \quad m, n=0,1,2, \cdots
$$

is bounded and continuous for $0 \leqq x, y<\infty$ [1, p. 344].

$$
\begin{aligned}
& \text { B. } \int_{0}^{\infty} G(x, y) d \mu(y)=1, \quad 0 \leqq x<\infty \quad[1, \text { p. 344] . } \\
& \text { C. } \lim _{N \rightarrow \infty} \int_{a}^{b} G_{N}(x, y) d \mu(y)=1, \quad 0 \leqq a<x<b \leqq \infty \\
& =0 \quad 0 \leqq a \leqq b<x<\infty \\
& =0 \quad 0<x<a \leqq b \leqq \infty[1, \mathrm{p}, 345] \text {. }
\end{aligned}
$$

D. With $x$ fixed, in $0 \leqq x<\infty$

$$
G(x, y)=C e^{-a_{1} y} y^{m_{1}-r} \oint\left(a_{1} x\right)\left[1+0\left(\frac{1}{y}\right)\right], \quad y \rightarrow \infty
$$

where $\mathrm{C}>0,[1, \mathrm{p} .348] . \quad m_{1}$ and $\oint(x)$ are defined in a way similar to that defined by D. T. Haimo. When $N$ is fixed, (11), (12) and (14) also hold for $G_{N}(x, y)$.

Lemma 2. With $x$ fixed in $0 \leqq x<\infty$

$$
\begin{aligned}
\frac{\partial^{r} G(x, y)}{\partial x^{r}}=C e^{-a_{1} y} y^{m_{1}-r} a_{1}^{r} \oint^{(r)}\left(a_{1} x\right)\left[1+0\left(\frac{1}{y}\right)\right] & , y \rightarrow \infty \\
r & =0,1,2,3, \cdots .
\end{aligned}
$$

Proof. For $r=0$ see (14); we will deal exclusively with the case where $r>0$. Let $a$ be a positive quantity such that $a_{1}<a<a_{2}$. One can readily show by a techique similar to that of D. T. Haimo that

$$
\begin{aligned}
G(x, y) & =y^{1 / 2-\gamma}\left[\left(\frac{d}{d a}\right)^{m_{1}}\left\{\psi(a) \oint(a x) K_{\gamma-1 / 2}(a y)\right\}\right]_{a=a_{1}} \\
& +y^{1 / 2-r} \int_{-\infty+a i}^{\infty+a i} \frac{z^{1 / 2+\gamma} J(x z) H_{\gamma-1 / 2}^{(1)}(y z) d z}{E(z)} .
\end{aligned}
$$

$1+m_{1}$ is the multiplicity of the zero of $E(z)$ at $z= \pm a_{1} i[1, \mathrm{pp}$. 347-349]. Therefore,

$$
\begin{aligned}
\frac{\hat{o}^{r} G(x, y)}{\partial x^{r}} & =y^{1 / 2-\gamma}\left[\left(\frac{d}{d a}\right)^{m_{1}}\left\{\psi(a) a^{r} \oint^{(r)}(a x) K_{r-1 / 2}(a y)\right\}\right]_{a=a_{1}} \\
& +y^{1 / 2-\gamma} \int_{-\infty+a i}^{\infty+a i} \frac{z^{1 / 2+\gamma+r} J^{(r)}(x z) H_{\gamma-1 / 2}^{(1)}(y z) d z}{E(z)}
\end{aligned}
$$


Differentiation within the integral sign in (17) is justified by virtue of [6, pp. 197-203] and it is also proved thereby that the aforesaid integral is $0\left[y^{-1 / 2} e^{-a y}\right], y \rightarrow \infty$ uniformly for $x$ lying in any compact subset of $I$. This result coupled with [1, Corollary 2.2, p. 347] establishes (15). This asymptotic order can also be established for $G_{N}(x, y)$ with fixed $N$ where $a_{1}$ will be replaced by $a_{N}$.

Lemma 3. Let $\Delta_{x}, G(x, y)$ and $G_{N}(x, y)$ be defined precisely in a way similar to that of D. T. Haimo. Then;

$$
\Delta_{k}^{(n)} G(x, y)=A_{0} G(x, y)+A_{1} G_{1}(x, y)+A_{2} G_{2}(x, y)+\cdots A_{n} G_{n}(x, y) \text {. }
$$

where $A_{0}, A_{1}, A_{2}, \cdots A_{n}$ are all polynomials of degree $2 n$ each in $a_{0}, a_{1}, a_{2}, \cdots a_{n}$.

Proof.

$$
\begin{aligned}
\Delta_{x} G(x, y) & =\int_{0}^{\infty} \frac{\left(-t^{2}\right) J(x t) J(y t) d \mu(t)}{E(t)} \quad[1, \text { p. 334] } \\
& =-\int_{0}^{\infty} \frac{\left[\frac{t^{2}}{a_{1}^{2}}+1-1\right] a_{1}^{2} J(x t) J(y t) d \mu(t)}{E(t)}
\end{aligned}
$$

or

$$
\Delta_{x} G(x, y)=a_{1}^{2}\left[G(x, y)-G_{1}(x, y)\right] .
$$

In the same way we can show that

$$
\Delta_{x} G_{m}(x, y)=a_{m+1}^{2}\left[G_{m}(x, y)-G_{m+1}(x, y)\right] .
$$

Using the technique of induction and operating by $\Delta_{x}$ repeatedly on $G(x, y),(18)$ follows readily in view of (19) and (20).

THeorem 1. Let $f \in \mathscr{Y}^{\prime}(I)$ and define $F(x)$ by

$$
F(x)=\left\langle\mu^{\prime}(y) f(y), G(x, y)\right\rangle=\left\langle f(y), \mu^{\prime}(y) G(x, y)\right\rangle .
$$

Then,

$$
F^{(m)}(x)=\left\langle\mu^{\prime}(y) f(y), \frac{\partial^{m}}{\partial x^{m}} G(x, y)\right\rangle, \quad m=1,2,3, \cdots .
$$

Proof. In this theorem and from now on $c$ is assumed to be $<a_{1}$. In view of (1), (15) and (18), $\mathrm{G}(x, y) \in \mathscr{G}(I)$ for a fixed $x$. Therefore, by (6) $\mu^{\prime}(y) G(x, y) \in \mathscr{l}(I)$, and as such (21) is meaningful. For a similar reason (22) also has meaning. Now, 
(23)

$$
\frac{F(x+\Delta x)-F(x)}{\Delta x}-\left\langle\mu^{\prime}(y) f(y), \frac{\partial G(x, y)}{\partial x}\right\rangle=\left\langle\mu^{\prime}(y) f(y), \theta_{\Delta x}(y)\right\rangle
$$

where

$$
\theta_{\Delta x}(y)=\frac{G(x+\Delta x, y)-G(x, y)}{\Delta x}-\frac{\partial G}{\partial x}(x, y) .
$$

We will prove the result only for $m=1$, but the proof for any positive integral values of $m$ can be given very simply by induction. Our objective will therefore, be achieved if we can show that

$$
\gamma_{k}\left[\theta_{J x}(y)\right] \rightarrow 0 \text { as } \Delta x \rightarrow 0 \text { for a fixed } y
$$

where $k=0,1,2,3, \cdots$.

In view of (18), it is enougn to show that

$$
\sup _{0<y<\infty}\left|e^{c y}\left[\frac{G_{n}(x+\Delta x, y)-G_{n}(x, y)}{\Delta x}-\frac{\partial}{\partial x} G_{n}(x, y)\right]\right| \rightarrow 0
$$

$$
\text { as } \Delta x \rightarrow 0
$$

for a fixed $x$ and $n$ where $n$ assumes values $0,1,2, \ldots$ Again using (15), (24) can be readily proved by the technique precisely similar to followed by Zemanian in [7, Th. 4.1]. This completes the proof of Theorem 7 .

Lemma 4. Let $\phi(x) \in D(I)$ and assume that

$$
\mathcal{J}_{x}^{(k)} \dot{\phi}(x)=\psi_{k}(x) \text {. }
$$

Then

$$
e^{c y} \int_{0}^{\infty} G_{N}(x, y)\left[\psi_{k}(y)-\psi_{k}(x)\right] d \mu(x) \stackrel{\Delta}{=} I \rightarrow 0
$$

uniformly on $0<y<\infty$ as $N \rightarrow \infty$.

Proof. We break up the integration in (26) into integrations on $0<x<y-\delta, \quad y-\delta<x<y+\delta$ and $y+\delta<x<\infty \quad(\delta>0)$, and denote the corresponding quantities by $I_{1}, I_{2}$ and $I_{3}$ respectively. By (25) $\dot{\psi}_{k}(x) \in D(I)$. Now

$$
I_{2}=e^{c y} \int_{y-\hat{o}}^{y+\tilde{o}} G_{N}(x, y)\left[\psi_{k}(y)-\psi_{k}(x)\right] d \mu(x) .
$$

By virtue of (12) and (27)

$$
\begin{aligned}
\left|I_{2}\right| & \leqq \sup _{y-\delta<x<y+\delta}\left|e^{c y}\left(\psi_{k}(y)-\psi_{k}(x)\right)\right| \\
& \leqq e^{c y} \delta \sup _{y-\delta<<\tau<y+\delta}\left|\psi_{k}^{(1)}(\tau)\right| .
\end{aligned}
$$


Since $\psi_{k}(x) \in D(I)$, the last quantity is bounded by $\delta B$ where $B$ is a constant with respect to $\delta$ and $y$ when $\delta$ is restricted to $0<\delta<1$. Therefore, given an $\varepsilon>0,\left|I_{2}\right|$ is bounded by $\varepsilon$ for $\delta=\min (1, \varepsilon / B)$. Fix $\delta$ this way. Next consider,

$$
\begin{aligned}
I_{1} & =e^{c y} \int_{0}^{y-\hat{\delta}} G_{N}(x, y)\left[\psi_{k}(y)-\psi_{k}(x)\right] d \mu(x) \\
& =e^{c y} \int_{0}^{y-\hat{\delta}} G_{N}(x, y) \psi_{k}(y) d \mu(x) \\
& -e^{c y} \int_{0}^{y-\delta} G_{N}(x, y) \psi_{k}(x) d \mu(x) .
\end{aligned}
$$

Let the support of $\dot{\phi}(x)$ be contained in $[A, B]$ where $0<A<B$. Therefore, the support of $\psi_{k}(x)$ will be also contained in $[A, B]$. Suppose that,

$$
\sup _{0<x<\infty}\left|e^{c x} \psi_{k}(x)\right|=M
$$

and

$$
\sup _{0<x<\infty}\left|\psi_{k}(x)\right|=m
$$

then

$$
\begin{aligned}
\left|I_{1}\right| & \leqq M \int_{0}^{y-\delta} G_{N}(x, y) d \mu(x)+e^{c y} \int_{0}^{y-\delta} G_{N}(x, y)\left|\psi_{k}(x)\right| d \mu(x) \\
& \leqq M \int_{\delta}^{\infty} G_{N}(u) d \mu(u)+e^{c y} \int_{0}^{y-\delta} G_{N}(x, y)\left|\psi_{k}(x)\right| d \mu(x) .
\end{aligned}
$$

It is a fact that

$$
\int_{0}^{\infty} G_{N}(u) d \mu(u)=2 \sum_{k=N+1}^{\infty} a_{k}^{-2} \rightarrow 0 \quad \text { as } N \rightarrow \infty \quad[1, \text { pp. 345-46] . }
$$

Again, in a way similar to what is done in (14) one can show that

$$
G_{N}(x, y)=C e^{-a_{N} y} y^{m_{1}-r} \oint\left(a_{.} x\right)\left[1+0\left(\frac{1}{y}\right)\right] .
$$

Since $c<a_{1} \leqq a_{N}$, we can choose a fixed quantity $k>B$ so large that the asymptotic estimate (29) is valid for $y>k$. Therefore, $e^{c y} G_{N}(x, y) \rightarrow$ 0 as $N \rightarrow \infty$ uniformly with respect to $x$ lying in any compact subset of $I$ and $y>k$. Now,

$$
\begin{aligned}
e^{c y} \int_{0}^{y-\delta} G_{N}(x, y)\left|\psi_{k}(x)\right| d \mu(x) & \leqq \mu^{\prime}(B) m \int_{A}^{B} e^{c y} G_{N}(x, y) d x \\
& \rightarrow 0 \text { uniformly for all } \\
y>k \text { as } & N \rightarrow \infty
\end{aligned}
$$


When $0<y \leqq k$ we have

$$
\begin{aligned}
e^{c y} \int_{0}^{y-\delta} G_{N}(x, y) \psi_{k}(x) d \mu(x) & \leqq m\left[\sup _{0<y<k} e^{c y}\right] \int_{0}^{y-\delta} G_{N}(x, y) d \mu(x) \\
& \rightarrow 0 \text { as } N \rightarrow \infty \text { uniformly }
\end{aligned}
$$

for all $0<y \leqq k,\left[1\right.$, p. 346]. Therefore, $\left|I_{1}\right| \rightarrow 0$ as $N \rightarrow \infty$ uniformly for all $y>0$. Again

$$
\begin{gathered}
I_{3}=e^{c y} \int_{y+\delta}^{\infty} G_{N}(x, y)\left[\psi_{k}(y)-\psi_{k}(x)\right] d \mu(x) . \\
\text { If } \mathrm{y}>B \text { then } I_{3}=0 .
\end{gathered}
$$

Therefore, we will now consider the case

$$
\begin{gathered}
y \leqq B . \\
I_{3}=e^{c y} \int_{y+\delta}^{\infty} G_{N}(x, y) \psi_{k}(y) d \mu(x)-e^{c y} \int_{y+\delta}^{\infty} G_{N}(x, y) \psi_{k}(x) d \mu(x) \\
\left|I_{3}\right| \leqq M \int_{y+\delta}^{\infty} G_{N}(x, y) d \mu(x)+m\left[\sup _{0<y<B} e^{c y}\right] \int_{y+\delta}^{\infty} G_{N}(x, y) d \mu(x) \\
\leqq\left[M+m \sup _{0<y \leqq B} e^{c y}\right] \int_{\delta}^{\infty} G_{N}(u) d \mu(u) \quad[1, \text { p. } 346] \\
\rightarrow 0 \text { as } N \rightarrow \infty \quad \text { uniformly for all } \quad 0<y \leqq B .
\end{gathered}
$$

In view of (30) therefore, we have proven that $\left|I_{3}\right| \rightarrow 0$ as $N \rightarrow \infty$. uniformly for all $y>0$. Altogether we have proven that

$$
\varlimsup_{N^{\prime} \rightarrow \infty}|I| \leqq \varepsilon \quad \delta<y<\infty .
$$

When $\mathrm{y} \leqq \delta$ we break up the integration in (26) into integrations on $0<x<y+\delta, y+\delta<x<\infty$ and represent the corresponding quantities. as $J_{1}$ and $J_{2}$ respectively.

Clearly $J_{2} \rightarrow 0$ as $N \rightarrow \infty$ uniformly for all $y>0$. Now,

$$
\begin{aligned}
\left|J_{1}\right| & \leqq e^{c \delta} \int_{0}^{y+\delta} G_{N}(x, y)\left|\psi_{k}(y)-\psi_{k}(x)\right| d \mu(x) \\
& \leqq e^{\alpha_{1}} \frac{\varepsilon}{B} \sup _{0<\tau<\infty}\left|\psi_{k}^{(1)}(\tau)\right|=\varepsilon^{\prime} \quad \text { (say) }
\end{aligned}
$$

$(30 \mathrm{~b})$

$$
\therefore \quad \varlimsup_{N \rightarrow \infty}|I| \leqq \varepsilon^{\prime} \quad \text { for all } 0<y \leqq \delta .
$$

Let $\eta=\operatorname{Max}\left[\varepsilon, \varepsilon^{\prime}\right]$. Note that by virtue of the fact that $\varepsilon$ is: arbitrary $\eta$ is also arbitrary. Combining (30a) and (30b) we have proven the fact that

$$
\varlimsup_{U^{\prime} \rightarrow \infty}|I| \leqq \eta, \quad 0<y<\infty
$$


Since $\eta$ is arbitrary, our lemma is proven.

We are now ready to prove the main theorem of this paper.

THEOREM 2. Let $f \in \mathscr{Y}^{\prime}(I)$ and let $F(x)$ be defined as in (21). Then for $\phi(x) \in D(I)$

$$
\left\langle\mu^{\prime}() P_{N}\left(\Delta_{x}\right) F(x), \phi(x)\right\rangle \rightarrow\left\langle\mu^{\prime} f, \phi\right\rangle \quad \text { as } N \rightarrow \infty
$$

where

$$
P_{N}\left(\Delta_{x}\right)=\prod_{k=1}^{N}\left(1-\frac{\Delta_{x}}{a_{k}^{2}}\right)
$$

Proof. The theorem will be proven by justifying steps in the following manipulations.

$$
\begin{aligned}
\left\langle\mu^{\prime}(x) P_{N}\left(\Delta_{x}\right) F(x), \phi(x)\right\rangle & =\left\langle\mu^{\prime}(x) P_{N}\left(A_{x}\right)\left\langle\mu^{\prime}(y) f(y), G(x, y)\right\rangle, \phi(x)\right\rangle \\
& =\left\langle\mu^{\prime}(x)\left\langle\mu^{\prime}(y) f(y), P_{n}\left(\Delta_{x}\right) G(x, y)\right\rangle, \phi(x)\right\rangle \\
& =\left\langle\mu^{\prime}(x)\left\langle\mu^{\prime}(y) f(y), G_{N}(x, y)\right\rangle, \phi(x)\right\rangle \\
& =\left\langle\mu^{\prime}(y) f(y),\left\langle\mu^{\prime}(x) G_{N}(x, y), \phi(x)\right\rangle\right\rangle \\
& \rightarrow\left\langle\mu^{\prime}(y) f(y), \phi(y)\right\rangle \quad \text { as } N \rightarrow \infty .
\end{aligned}
$$

The fact that (31) is true follows at once in view of (21), and that the expressions in (31) are each equal to (32) is an immediate consequence of (21) and (22). That (32) is equal to (33) follows by [1; Lemma 4.1, p. 360]. relation

To prove that (33) and (34) are equal we need show the following

$$
\begin{aligned}
& \int_{A}^{B}\left\langle\mu^{\prime}(y) f(y), G_{N}(x, y)\right\rangle \phi(x) d \mu(x) \\
& \quad=\left\langle\mu^{\prime}(y) f(y), \int_{A}^{B} G_{N}(x, y) \dot{\phi}(x) d \mu(x)\right\rangle .
\end{aligned}
$$

As before we assume that the support of $\phi(x)$ is contained in $[A, B]$ where $0<A<B$.

In view of asymptotic orders of $G_{m}(x, y),(m=0,1,2,3, \cdots)$ for fixed $x$ and large $y$ as stated in (15) and using the technique of the Riemann sum, (36) is proved in a way exactly similar to that employed in [4, Th. 2]. Note that (18) is also to be made use of in proving the assertion in (36).

We now proceed to prove that (34) is equal to (35). Our objective will be fulfilled if we can show that

$$
\left\langle\mu^{\prime}(x) G_{N}(x, y), \phi(x)\right\rangle \rightarrow \phi(y) \text { in } \mathscr{C}(I) \quad \text { as } N \rightarrow \infty \text {. }
$$

Now, 


$$
\left\langle\mu^{\prime}(x) G_{N}(x, y), \phi(x)\right\rangle=\int_{A}^{B} G_{N}(x, y) \phi(x) d \mu(x)
$$

and

$$
\Delta_{y}^{(k)}\left\langle\mu^{\prime}(x) G_{N}(x, y), \phi(x)\right\rangle=\int_{A}^{B}\left\{\Delta_{y}^{(k)} G_{N}(x, y)\right\} \dot{\phi}(x) d \mu(x) .
$$

By using (15) the differentiation process within the sign of integration in (38) is easily justified. Now,

$$
\Delta_{x} G_{N}(x, y)=\Delta_{y} G_{N}(x, y)=\int_{0}^{\infty} \frac{\left(-t^{2}\right) J(x t) J(y t) d \mu(t)}{E_{N}(t)} \quad[1 ; \text { p. 334]!. }
$$

Again

$$
\begin{aligned}
\int_{A}^{B}\left\{\Delta_{x} G_{N}(x, y)\right\} \dot{\phi}(x) d \mu(x)= & \int_{A}^{B} G_{N}(x, y)\left\{\Delta_{x} \dot{\phi}(x)\right\} d \mu(x) \\
& {[\text { integration by parts] }} \\
= & \int_{0}^{\infty} G_{N}(x, y)\left\{\Delta_{x} \dot{\phi}(x)\right\} d \mu(x) .
\end{aligned}
$$

Therefore by repeatedly using (39) and (40) one can readily show that

$$
\begin{aligned}
& \int_{A}^{B}\left\{\Delta_{y}^{(k)} G_{N}(x, y)\right\} \phi(x) d \mu(x)=\int_{0}^{\infty} G_{N}(x, y)\left\{\Delta_{x}^{(k)} \phi(x)\right\} d \mu(x) \\
& \therefore \quad e^{c y} \Delta_{y}^{(k)}\left[\left\langle\mu^{\prime}(x) G_{N^{N}}(x, y), \phi(x)\right\rangle-\phi(y)\right] \\
& \quad=e^{c y}\left[\int_{0}^{\infty} G_{\Lambda}(x, y)\left(\left\{\Delta_{x}^{(k)} \phi(x)\right\}-\left\{\Delta_{y}^{(k)} \phi(y)\right\}\right) d \mu(x)\right. \\
& \quad=e^{c y}\left[\int_{0}^{\infty} G_{N^{\prime}}(x, y)\left\{\psi_{k}(x)-\psi_{k}(y)\right\} d \mu(x)\right] \\
& \quad \rightarrow 0 \text { uniformly on } 0<y<\infty \text { as } N \rightarrow \infty
\end{aligned}
$$

in view of Lemma 4 . Thus the proof of Theorem 2 is complete.

The author expresses his gratitude to Professor Zeev Ditzian for his valuable suggestions.

\section{REFERENCES}

1. D. T. Haimo, Integral equations associated with Hankel convolutions, Trans. Amer. Math. Soc. 116 (1965), 330-375.

2. I. I. Hirschman, Jr., Variation diminishing Hankel transforms, J. D'Analyse Mathe'matique, Extrait Du Vol. VIII, Jerusalem, 1960/1961.

3. - and D. V. Widder, The Convolution transform, Princeton Univ. Press, Princeton, N.J., 1955.

4. J. N. Pandey and A. H. Zemanian, Complex inversion for the generalized convolution transformation, Pacific J. Math. 25 (1968) 147-157. 
5. L. Schwartz, Theorie des Distributions, Vols. I and II, Hermann, Paris, 1957 and 1959.

6. G. N. Watson, A Treatise on the theory of Bessel functions, Second Edition, 1962. 7. A. H. Zemanian, A generalized convolution transformation, J. Soc. Indust. and Appl. Math. 15 (1967).

8. - A distributional Hankel transformation, J. Soc. Indust. and Appl. Math. 14 (1966), 561-576.

9. - Distribution theory and transform analysis, McGraw-Hill Book Co., New York, 1965.

Received July 15, 1968. Part of this work was supported by the grant NRC A 5298 .

The University of Alberta

Edmonton, Alberta, Canada

Now at

Carleton University

Ottawa 1, Ontario, Canada 



\section{PACIFIC JOURNAL OF MATHEMATICS}

\section{EDITORS}

\author{
H. ROYDEN \\ Stanford University \\ Stanford, California \\ R. R Phelps \\ University of Washington \\ Seattle, Washington 98105
}

\author{
J. Dugundui \\ Department of Mathematics \\ University of Southern California \\ Los Angeles, California 90007
}

RICHARD ARENS

University of California

Los Angeles, California 90024

\section{ASSOCIATE EDITORS}
E. F. BeCKENBACH
B. H. NeUmanN
F. WolF
K. YosidA

\section{SUPPORTING INSTITUTIONS}

\author{
UNIVERSITY OF BRITISH COLUMBIA \\ CALIFORNIA INSTITUTE OF TECHNOLOGY \\ UNIVERSITY OF CALIFORNIA \\ MONTANA STATE UNIVERSITY \\ UNIVERSITY OF NEVADA \\ NEW MEXICO STATE UNIVERSITY \\ OREGON STATE UNIVERSITY \\ UNIVERSITY OF OREGON \\ OSAKA UNIVERSITY \\ UNIVERSITY OF SOUTHERN CALIFORNIA
}

\author{
STANFORD UNIVERSITY \\ UNIVERSITY OF TOKYO \\ UNIVERSITY OF UTAH \\ WASHINGTON STATE UNIVERSITY \\ UNIVERSITY OF WASHINGTON

$* * * * *$
AMERICAN MATHEMATICAL SOCIETY
CHEVRON RESEARCH CORPORATION
TRW SYSTEMS
NAVAL WEAPONS CENTER

The Supporting Institutions listed above contribute to the cost of publication of this Journal, but they are not owners or publishers and have no responsibility for its content or policies.

Mathematical papers intended for publication in the Pacific Journal of Mathematics should be in typed form or offset-reproduced, double spaced with large margins. Underline Greek letters in red, German in green, and script in blue. The first paragraph or two must be capable of being used separately as a synopsis of the entire paper. It should not contain references to the bibliography. Manuscripts, in duplicate if possible, may be sent to any one of the four editors. Please classify according to the scheme of Math. Rev. 36, 1539-1546. All other communications to the editors should be addressed to the managing editor, Richard Arens, University of California, Los Angeles, California, 90024.

50 reprints are provided free for each article; additional copies may be obtained at cost in multiples of 50 .

The Pacific Journal of Mathematics is published monthly. Effective with Volume 16 the price per volume (3 numbers) is $\$ 8.00$; single issues, $\$ 3.00$. Special price for current issues to individual faculty members of supporting institutions and to individual members of the American Mathematical Society: $\$ 4.00$ per volume; single issues $\$ 1.50$. Back numbers are available.

Subscriptions, orders for back numbers, and changes of address should be sent to Pacific Journal of Mathematics, 103 Highland Boulevard, Berkeley, California, 94708.

PUBLISHED BY PACIFIC JOURNAL OF MATHEMATICS, A NON-PROFIT CORPORATION

Printed at Kokusai Bunken Insatsusha (International Academic Printing Co., Ltd.), 7-17. Fujimi 2-chome, Chiyoda-ku, Tokyo, Japan. 


\section{Pacific Journal of Mathematics}

Vol. 28, No. 3

May, 1969

Jon F. Carlson, Automorphisms of groups of similitudes over $F_{3} \ldots \ldots \ldots$

W. Wistar (William) Comfort, Neil Hindman and Stelios A. Negrepontis,

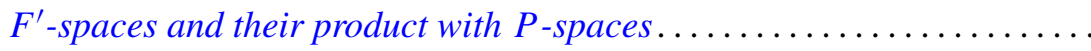

Archie Gail Gibson, Triples of operator-valued functions related to the unit

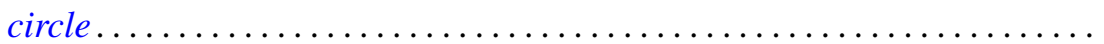

David Saul Gillman, Free curves in $E^{3}$

E. A. Heard and James Howard Wells, An interpolation problem for

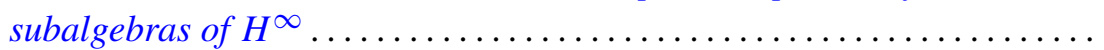

Albert Emerson Hurd, A uniqueness theorem for weak solutions of symmetric

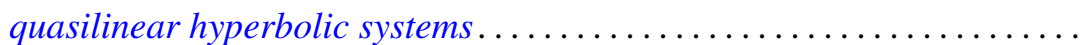

E. W. Johnson and J. P. Lediaev, Representable distributive Noether

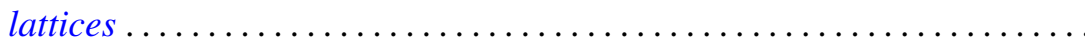

David G. Kendall, Incidence matrices, interval graphs and seriation in

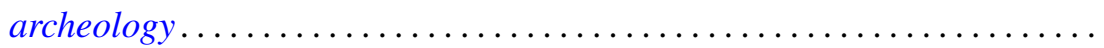
565

Robert Leroy Kruse, On the join of subnormal elements in a lattice ....... 571

D. B. Lahiri, Some restricted partition functions; Congruences modulo 3 .... 575

Norman D. Lane and Kamla Devi Singh, Strong cyclic, parabolic and conical differentiability........................................

William Franklin Lucas, Games with unique solutions that are

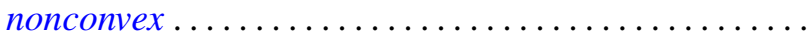

Eugene A. Maier, Representation of real numbers by generalized geometric series.

Daniel Paul Maki, A note on recursively defined orthogonal polynomials ...

Mark Mandelker, $F^{\prime}$-spaces and z-embedded subspaces ...

James R. McLaughlin and Justin Jesse Price, Comparison of Haar series with gaps with trigonometric series

Ernest A. Michael and A. H. Stone, Quotients of the space of irrationals ....

William H. Mills and Neal Zierler, On a conjecture of Golomb ...

J. N. Pandey, An extension of Haimo's form of Hankel convolutions ...

Terence John Reed, On the boundary correspondence of quasiconformal mappings of domains bounded by quasicircles...

Haskell Paul Rosenthal, A characterization of the linear sets satisfying Herz's criterion.

George Thomas Sallee, The maximal set of constant width in a lattice...

I. H. Sheth, On normaloid operators

James D. Stasheff, Torsion in BBSO ...

Billy Joe Thorne, A - P congruences on Baer semigroups.

Robert Breckenridge Warfield, Jr., Purity and algebraic compactness for

modules... 